\title{
Development of a Liquid Scintillator Using Water for a Next Generation Neutrino Experiment
}

\author{
Sun Heang So, Kyung Kwang Joo, Ba Ro Kim, Bong Keon Kim, Seung Chan Kim, \\ Chang Dong Shin, and In Sung Yeo \\ Department of Physics, Chonnam National University, Gwangju 500-757, Republic of Korea \\ Correspondence should be addressed to Kyung Kwang Joo; kkjoo@chonnam.ac.kr and Bong Keon Kim; funny369@naver.com
}

Received 13 August 2014; Accepted 9 October 2014; Published 27 October 2014

Academic Editor: Ashot Chilingarian

Copyright (c) 2014 Sun Heang So et al. This is an open access article distributed under the Creative Commons Attribution License, which permits unrestricted use, distribution, and reproduction in any medium, provided the original work is properly cited. The publication of this article was funded by $\mathrm{SCOAP}^{3}$.

\begin{abstract}
A feasibility study on a new type of liquid scintillator based on water has been performed. Mainly due to the differences in polarities between water and oil, organic solvents are not mixed in water. In order to make a liquid scintillator based on water, a surfactant that contains hydrophilic and hydrophobic groups is used. The surfactant keeps water and organic solvents apart in solution. Good physical and optical parameters and long-term stability are required to use liquid scintillator based on water in massive detector for the next generation neutrino experiments. In this paper, we report the characteristics and the possibility of liquid scintillators using water with various new surfactants.
\end{abstract}

\section{Introduction}

Liquid scintillator (LS) has been commonly used to detect neutrinos emitted from reactors through inverse beta decay process (IBD, $\left.\bar{v}_{e}+p \rightarrow e^{+}+n\right)$ followed by neutron capture [1]. The threshold energy of incident neutrinos for IBD is $1.8 \mathrm{MeV}$ [2]. A LS is a mixture of an organic base solvent and fluor. Fluor is used to achieve light emission. In addition, sometimes a secondary wavelength shifter (WLS), which is a fluorescent organic chemical material that absorbs photons and reemits photons at a longer wavelength, is added [3]. It shifts the wavelength to the optimal photomultiplier tube (PMT) sensitive region [4].

For organic solvents, pseudocumene $\left(\mathrm{PC}, \mathrm{C}_{9} \mathrm{H}_{12}\right)$, linear alkyl benzene (LAB, $\mathrm{C}_{n} \mathrm{H}_{2 n+1}-\mathrm{C}_{6} \mathrm{H}_{5}$, where $n=10 \sim 13$ ), phenylxylylethane (PXE, $\mathrm{C}_{16} \mathrm{H}_{18}$ ), and diisopropylnaphthalene (DIN, $\mathrm{C}_{16} \mathrm{H}_{20}$ ) are used [5-7]. Among them, PC-based LS has been widely used due to its high light yield (LY) compared to LSs using other organic base solvents [4]. However, PC has a low flashpoint $\left(46^{\circ} \mathrm{C}\right)$ and is very toxic to human body and environment. Since neutrino detector is usually located deep underground at the mountain region, strict safety regulations need to be satisfied [8]. Liquid handling of toxic PC is not easy for a large-scale detector. In addition, high cost generated by using PC-based LS cannot be avoided. Furthermore, some PC-based LSs have poor compatibility with detector materials [9].

Therefore, if a LS using water is developed and replaces a toxic liquid scintillator, it will be less hazardous, more useful, and environmentally friendly and the compatibility problem can be solved. Usually a scale of next generation neutrino detector will be a few or hundred thousand tons [10, 11]. Mass production of a liquid scintillator using water will be much easier than using oil-based solvents. In addition, due to the mixture usage of water and the scintillation light of liquid scintillator, we can go lower Cherenkov energy threshold, so that a low energy neutrino can be reconstructed. However, in order to use a liquid scintillator based on water for a neutrino detector, it should provide a sufficient LY and also have a long attenuation length and chemical stability over several years of experiments. In order to synthesize a liquid scintillator using water, it is essential to dissolve organic liquid 
scintillator into water. The synthesis process mainly takes place in water. During all synthesis processes, $18 \mathrm{M} \Omega$ highpure water produced by Millipore Milli-Q water system is used. It is well known that an attenuation length of highpurity water is very large [12-14]. The surfactant is to emulsify the organic liquid scintillator into the water.

For this study, samples are classified into three groups. The first sample is made by mixing just small portion of LS and water with a help of surfactant. We name this sample as a water-based liquid scintillator (WbLS, S1 group). For a base organic solvent, LAB is used because it is safe and LY is comparable to that of $\mathrm{PC}[7,15]$. It is essential to have a sufficient concentration of LS in water for WbLS. Some preliminary results were reported by BNL group in WbLS case [16]. The second is that just a fluor is dissolved into pure water (S2 group). Namely, a fluor is added in water Cherenkov detector to shift the wavelength into a longer one. Several wavelength shifters for a Cherenkov detector were tested by SNO group [17]. The last one is that a fluor is added to water using different surfactants (S3 group). A fluor is directly dissolved into surfactant at room temperature, which makes a solution. Then this solution is slowly diluted with pure water to the desired concentration. A possibility was reported 30 years ago [18]. Therefore, we have developed several promising candidates using possible different surfactants. This is a major motivation of this paper.

For a fluor, PPO $\left(\mathrm{C}_{15} \mathrm{H}_{11} \mathrm{NO}, 2,5\right.$-diphenyloxazole, neutrino grade), Carbostyril $124\left(\mathrm{C}_{10} \mathrm{H}_{10} \mathrm{~N}_{2} \mathrm{O}\right.$, 7-amino-4-methyl-2(1H)-quinolinol, CS-124), and Alexa fluor 350 (AF-350) were dissolved. AF-350 is a water soluble blue-fluorescent dye. $\mathrm{CS}-124$ and $\mathrm{AF}-350$ are $\mathrm{pH}$-insensitive fluor. BisMSB $\left\{\left(\mathrm{CH}_{3} \mathrm{C}_{6} \mathrm{H}_{4} \mathrm{CH}=\mathrm{CH}\right)_{2} \mathrm{C}_{6} \mathrm{H}_{4}, \quad\right.$ 1,4-bis(2-methylstyryl) benzene, scintillation grade $\}$ is also used as a secondary WLS. Fluor concentration is determined by optimizing the maximum LY of all samples. In addition, attenuation length and chemical stability are also considered. In this paper, we briefly describe the material chemistry, preparation, and report on physical and optical properties of these three sample groups.

\section{Choice of Various Surfactants}

Water and organic solvent are not mixed with each other and will quickly separate into two layers. In order to reduce the tension between polar (hydrophilic) surface and nonpolar (hydrophobic) surface, a surfactant is needed. Currently a few hundred surfactants exist [19]. Therefore, finding a suitable surfactant plays a key role in synthesizing liquid scintillators using water. The structure of a surfactant and micelles is shown in Figure 1. A surfactant consists of a polar hydrophilic group and a nonpolar hydrophobic group. Surfactant molecules organize to form micelles; namely, micelle structure is a special structure constructed by a surfactant. The hydrophilic "head" regions are in contact with surrounding $\mathrm{H}_{2} \mathrm{O}$, and the hydrophobic tail forms the middle of a micelle around LS. The organic LS is dissolved in this tail region and the polar heads of surfactant molecules dissolve the micelle in the water.
One of surfactant candidates is LAS, which is a sulfonicacid (HO-S-O-OH) derivative of alkyl benzene [16]. It is very safe to use. LAS has an alkyl chain and a benzene group which acts as a solvent to extract oils (lipophilic), while the attached sulfonate group is soluble in water (hydrophilic). Therefore, it acts as surfactant and LS simultaneously. According to BNL group study, LY of WbLS based on LAS can be extended up to several tens percentages of ordinary LS [16]. However, the optical property of the prepared $\mathrm{WbLS}$ material does not yet meet the requirements for a large detector [16]. Purification processes are to be applied. In addition, LAS has dark brown color. Additional work to remove color is needed. Furthermore, previous WbLS trials were not satisfactory because it is either gel-like or unstable over time [20].

For other candidates we tried Triton X (ethoxylated octylphenol), Pluronic (Poloxamer), Tween (Polysorbate), IGEPAL CO-630, RMA, GOE, NP-10, coco-betaine, POE (60) hydrogenated castor oil (HCO-60), and so forth, which are commercially available. Table 1 shows a summary of several surfactants used. General synthesis process of S1 sample is shown in Figure 2. First, surfactant is added to water. Then, in order to make a master solution, proper quantity of PPO and bis-MSB is dissolved in an organic solvent. Finally a master solution is mixed with a solution of water and surfactant under moderate speed of impeller. In the case of S2 sample, a surfactant is not necessary. Therefore, CS-124 or AF-350 can be directly dissolved into water. For CS-124 or AF-350 case, an order of a few ppm level is added [17]. In order to make S3 sample, we have tested several surfactants as shown in Table 2. In most of the S3 sample, a tint color has developed as time elapses. Furthermore, some S3 samples go into two phases in a few months. Therefore, HCO-60, Triton $\mathrm{X}-100$, and Polysorbate- 80 plus coco-betaine are selected as main surfactants and the quantity used is optimized, based on LY measurement. Unlike other surfactants, initial state HCO60 is solid, so that dissolving order affects the result. HCO60 and PPO are mixed first and then heated with a proper temperature until HCO-60 is melted down and mixed with PPO together.

\section{Characteristics of Liquid Scintillator Using Water}

3.1. Light Yield (LY). Above all, in order to use a liquid scintillator using water for the neutrino experiments, they provide enough light emission. First, samples were illuminated by an ultraviolet (UV, $\sim 250 \mathrm{~nm}$ ) lamp. At least we can clearly see that light is emitted from S1, S2, and S3 samples, as shown in Figure 3. For more detailed inspection, a test setup for data acquisition (DAQ) is prepared as shown schematically in Figure 4 . We made a Teflon cylindrical tube $12 \mathrm{~cm}$ in diameter and $5 \mathrm{~cm}$ in length. The volume of a container is approximately $500 \mathrm{~mL}$. 5-inch-diameter Hamamatsu H6527 PMTs are attached to both sides of the glass in the cylindrical Teflon tube. This PMT has a bialkali photocathode whose spectral response is maximum at around wavelength of $420 \mathrm{~nm}$ with a $\sim 23 \%$ quantum efficiency. The anode pulse signal was fed into 


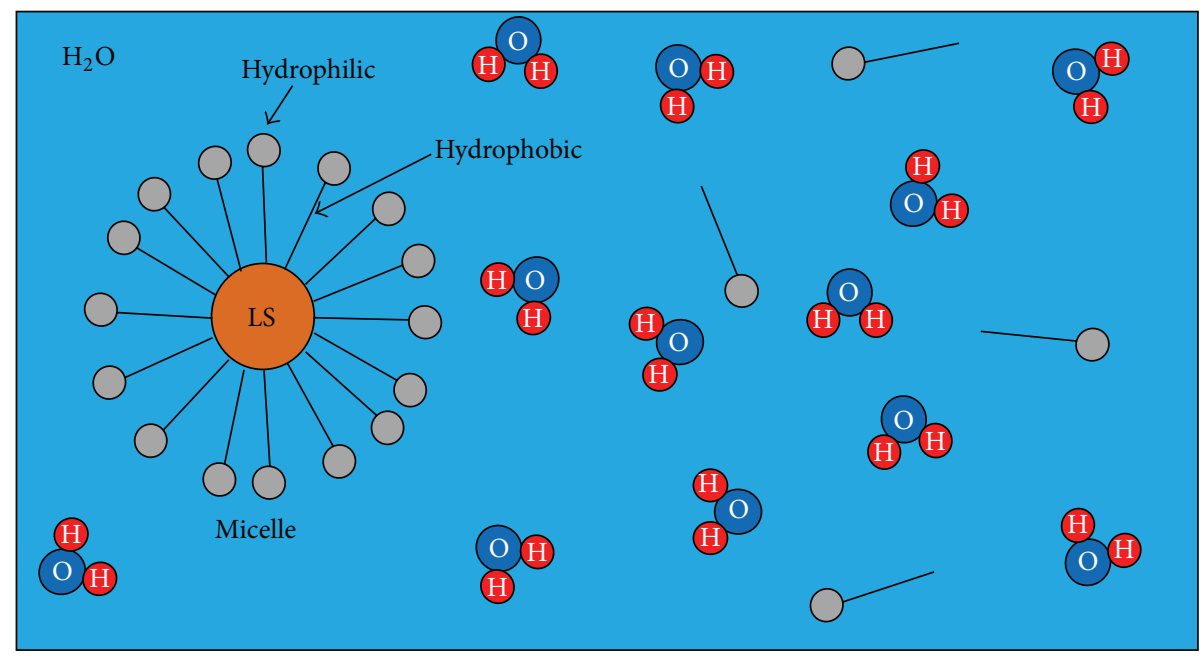

FIGURE 1: Structure of surfactant and micelle in a LS. Surfactant is a material having hydrophilic group and hydrophobic (or lipophilic) group. Surfactant molecules organize to form micelles. It helps dissolve LS in water.
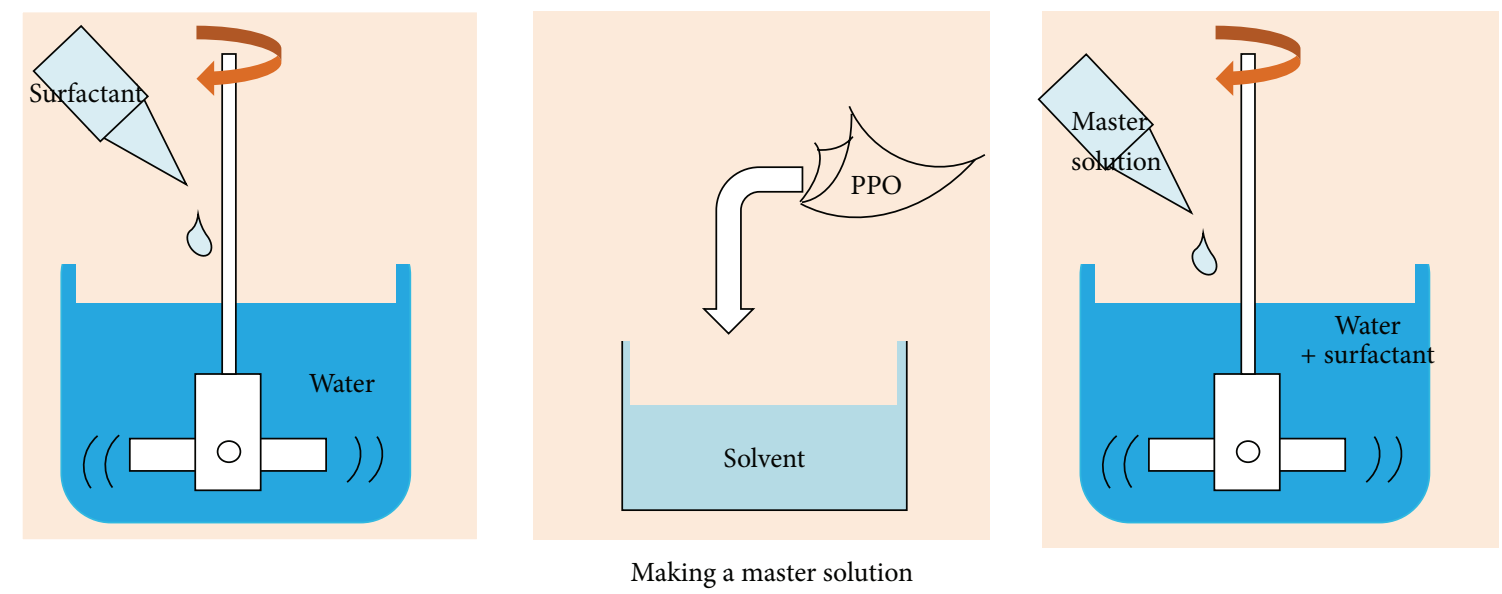

FIGURE 2: Synthesis process of WbLS (S1 sample). Surfactant is added to water. PPO is dissolved in organic solvent. Finally master solution is mixed with solution of water and surfactant with a proper speed of impeller.

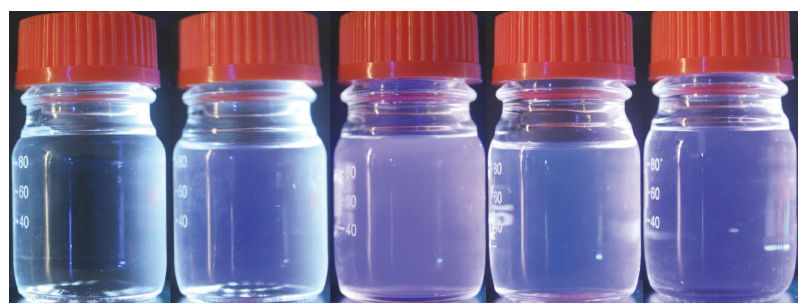

FIGURE 3: Light is emitted from samples using $250 \mathrm{~nm}$ wavelength UV lamp. From left, pure water $\left(\mathrm{H}_{2} \mathrm{O}\right)$, S1 (Triton X-100), S2 (CS124), S3 (HCO-60), and LAB-based LS. Pure water does not emit light. LAB-based LS is shown for the comparison of light intensity with other samples.

a homemade $400 \mathrm{MHz}$ flash analog digital converter (FADC) module. The data acquisition system (DAQ) is composed of VME crate and FADC $400 \mathrm{MHz}$ data processing modules and a PC running ROOT software.
The energy spectrum was measured and then fitted by using the proper functions. Sufficient LY is essential to reconstruct the energies of the events. The LY of the samples was determined by using the full width at half maximum (FWHM) of the Compton scattering edge position. $\mathrm{A}^{137} \mathrm{Cs}$ $(0.662 \mathrm{MeV})$ source is used. The LY of several samples is summarized in Figure 5. In all S1, S2, and S3 samples, we have roughly $\sim 10 \%$ of LY relative to that of a $100 \%$ LABbased LS. Even though the level of $\sim 10 \%$ output is not high enough, we need to further improve and compensate this deficit. However, at least a liquid scintillator using water can be feasible as a next generation neutrino detector.

3.2. Transmittance ([T]) and Attenuation Length $\left(\lambda_{\text {att }}\right)$. A liquid scintillator using water should be optically transparent if it is to be used as a massive next generation neutrino detector. After synthesizing various samples as listed in Table 3 , eye inspection was routinely performed over $\sim$ one 
TABLE 1: Several surfactants used for synthesizing a LS using water.

\begin{tabular}{|c|c|c|c|c|}
\hline Surfactants & Commercial name & Formular & Color & Type \\
\hline Linear alkyl sulfonic-acid (HO-S-O-OH) & LAS & $\mathrm{RC}_{6} \mathrm{H}_{4} \mathrm{SO}_{3} \mathrm{Na}$ & Dark brown & Anionic \\
\hline $\begin{array}{l}\text { Ethoxylated octylphenol } \\
\text { (iso-octyl phenoxy polyethoxy ethanol) }\end{array}$ & Triton X-100 & $\begin{array}{l}\mathrm{C}_{32} \mathrm{H}_{56} \mathrm{O}_{10} \\
\mathrm{C}_{34} \mathrm{H}_{60} \mathrm{O}_{11} \\
\end{array}$ & White & Nonionic \\
\hline $\begin{array}{l}\text { Polyoxyethylene (80) sorbitan } \\
\text { monooleate }\end{array}$ & $\begin{array}{c}\text { Tween- } 80 \\
\text { Polysorbate- } 80\end{array}$ & $\mathrm{C}_{64} \mathrm{H}_{124} \mathrm{O}_{26}$ & Brown & Nonionic \\
\hline Sorbitan monooleate, sorbitan oleate & Span-80 & $\mathrm{C}_{24} \mathrm{H}_{44} \mathrm{O}_{6}$ & Light yellow & Nonionic \\
\hline Nonylphenol, branched, ethoxylated & IGEPAL CO-630 & $\begin{array}{l}\mathrm{C}_{33} \mathrm{H}_{60} \mathrm{O}_{10} \\
\mathrm{C}_{35} \mathrm{H}_{64} \mathrm{O}_{11} \\
\end{array}$ & White & Nonionic \\
\hline Nonylphenol ethoxylate 10 & NP-10 & $\mathrm{C}_{35} \mathrm{H}_{64} \mathrm{O}_{11}$ & White & Nonionic \\
\hline POE (60) hydrogenated castor oil & HCO-60 & $\mathrm{C}_{18} \mathrm{H}_{37} \mathrm{NO}_{3}$ & Opaque & Nonionic \\
\hline Cocamidopropyl betaine & Coco-betaine & $\mathrm{C}_{19} \mathrm{H}_{38} \mathrm{~N}_{2} \mathrm{O}_{3}$ & Light yellow & Ampholytic \\
\hline Sodium cocoyl glutamate & & $\mathrm{RC}_{6} \mathrm{H}_{7} \mathrm{NO}_{5} \mathrm{Na}$ & Light yellow & Anionic \\
\hline $\begin{array}{l}\text { Sodium polyacrylate } \\
\text { Hydrogenated polydecene } \\
\text { Trideceth- } 6\end{array}$ & $\begin{array}{l}\text { OptaSense } \\
\text { RMA-52 }\end{array}$ & $\begin{array}{c}\left.\mathrm{C}_{3} \mathrm{H}_{3} \mathrm{O}_{2} \mathrm{Na}\right]_{n} \\
\mathrm{C}_{10} \mathrm{H}_{20} \\
\mathrm{C}_{15} \mathrm{H}_{32} \mathrm{O}_{2} \\
\end{array}$ & White & Anionic \\
\hline Glyceryl oleate/elaidate & GOE & $\mathrm{C}_{39} \mathrm{H}_{72} \mathrm{O}_{6}$ & Opaque & Non ionic \\
\hline Sodium Peg-7 Olive oil carboxylate & OLIVEM-400 & & Light yellow & Anionic \\
\hline
\end{tabular}

TABLE 2: Several surfactants used for synthesizing a LS using water.

\begin{tabular}{|c|c|c|c|c|}
\hline Sample group & Surfactant & Fluor & Fluor concentration & Eye inspection (time elapses) \\
\hline \multirow{5}{*}{ S1 } & Triton X-100 & $\mathrm{PPO}$ & $\sim 0.1 \mathrm{~g} / 100 \mathrm{~mL}$ & Clear \\
\hline & NP-10 & $\mathrm{PPO}$ & $\sim 0.1 \mathrm{~g} / 100 \mathrm{~mL}$ & Clear \\
\hline & IGEPAL CO-630 & $\mathrm{PPO}$ & $\sim 0.1 \mathrm{~g} / 100 \mathrm{~mL}$ & Unclear \\
\hline & LAS & $\mathrm{PPO}$ & $\sim 0.1 \mathrm{~g} / 100 \mathrm{~mL}$ & Clear \\
\hline & Tween-80 & $\mathrm{PPO}$ & & PPO is not solved \\
\hline \multirow{2}{*}{ S2 } & & CS-124 & $\sim$ ppm & Clear \\
\hline & & AF-350 & $\sim$ ppm & Clear \\
\hline \multirow{8}{*}{ S3 } & HCO-60 & PPO & $\sim 0.1 \mathrm{~g} / 100 \mathrm{~mL}$ & Clear \\
\hline & Triton X-100 & $\mathrm{PPO}$ & $\sim 0.1 \mathrm{~g} / 100 \mathrm{~mL}$ & Clear \\
\hline & Polysorbate- 80 & $\mathrm{PPO}$ & $\sim 0.1 \mathrm{~g} / 100 \mathrm{~mL}$ & Clear \\
\hline & RMA-52 & $\mathrm{PPO}$ & & PPO is not solved \\
\hline & GOE & $\mathrm{PPO}$ & & PPO is not solved \\
\hline & Coco-betaine & $\mathrm{PPO}$ & $\sim 0.1 \mathrm{~g} / 100 \mathrm{~mL}$ & Clear \\
\hline & OLIVEM-400 & $\mathrm{PPO}$ & $\sim 0.1 \mathrm{~g} / 100 \mathrm{~mL}$ & Tint color \\
\hline & Polysorbate- $80+$ coco-betaine & $\mathrm{PPO}$ & $\sim 0.1 \mathrm{~g} / 100 \mathrm{~mL}$ & Clear \\
\hline
\end{tabular}

year. For more detailed inspection, a transmittance value was measured by means of spectrometric technique by using a Shimadzu UV-1800 UV-VIS spectrophotometer. The optical quality of samples is evaluated by measuring their light transmittance in the wavelength region between 200 and $600 \mathrm{~nm}$. The light transmittance value $([T])$ is defined as $T=I / I_{0}$, where $I_{0}$ and $I$ are the intensities of the incident and the emitted light, respectively. The absorption value, $A$, is related to $T$ as $A=-\log T$. This describes the distance over which the initial intensity $I_{0}$ is reduced by $e^{-1}[8,21]$. Figure 6 shows $[T]$ and $[A]$ values of several samples on one plot. The transmittance value, $[T]$, of S1 (Triton X-100) and S3 (HCO-60) samples is over $\sim 80 \%$ in the wavelength range above $400 \mathrm{~nm}$. In the case of S2 (CS-124), it is over 90\% from $350 \mathrm{~nm}$ to $600 \mathrm{~nm}$.

The attenuation length $\lambda_{\text {att }}$ was extracted from $\lambda_{\text {att }}=$ $0.4343 L / A$, based on the Beer-Lambert-Bouguer empirical law $[5,15,22]$. Here, $L$ is the path length of a cuvette cell, and $A$ is the absorption value at 420 or $430 \mathrm{~nm}$. This is the wavelength region where the main scintillation energy transfer mechanism occurs and where the Hamamatsu H6527 PMT is most sensitive. The measurement was carried out in a $10 \mathrm{~cm}$ path-length quartz cuvette cell. The attenuation length of S2 (CS-124) at a wavelength of $430 \mathrm{~nm}$ is more than $\sim 40 \mathrm{~m}$. However, in S1 (Triton X-100) and S3 (HCO-60) samples, we need to purify the quality of samples. 


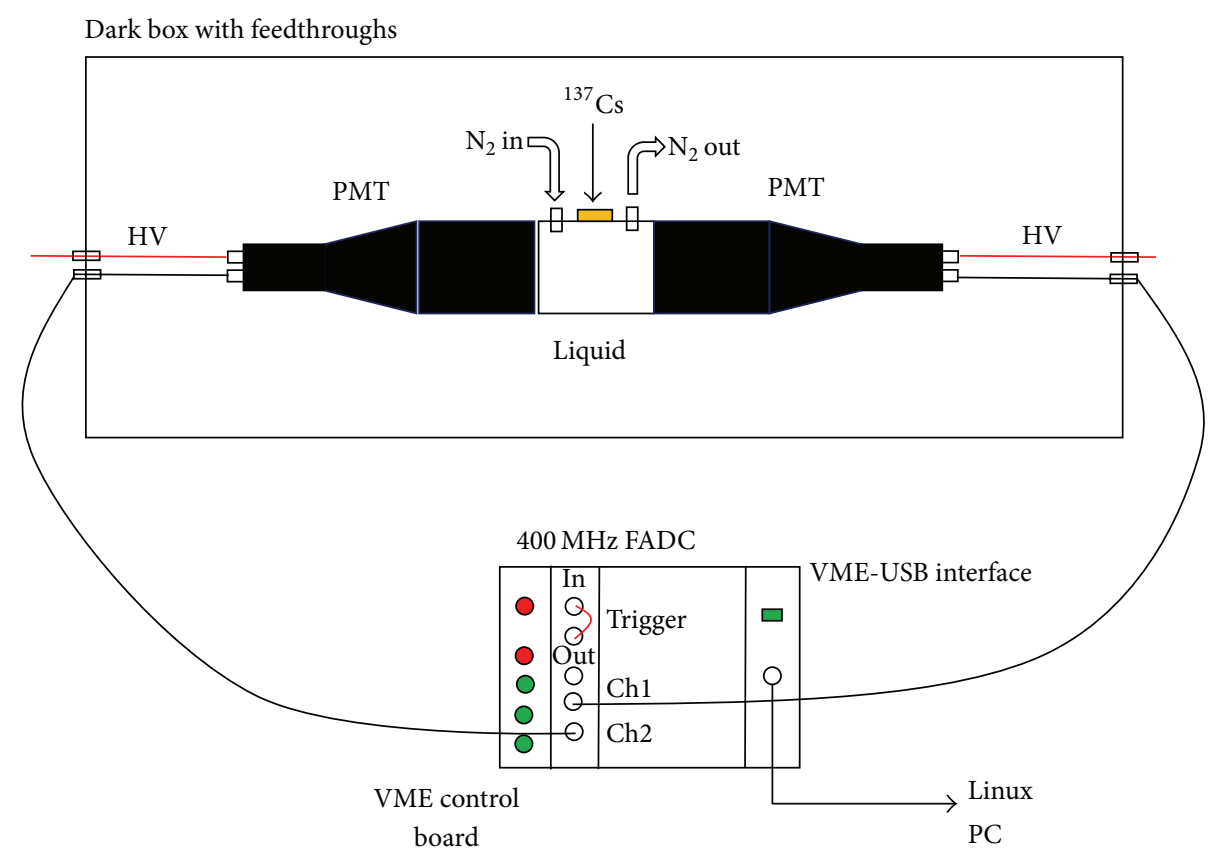

FIgURE 4: Schematic layout of the experimental test setup for DAQ. A homemade FADC-based DAQ system is designed. A self-trigger in the FPGA chip on the FADC module is used. A built-in discriminator with an adjustable $10 \mathrm{mV}$ threshold is applied. Data are transferred to Linux-based operating computer through VME-USB interface.

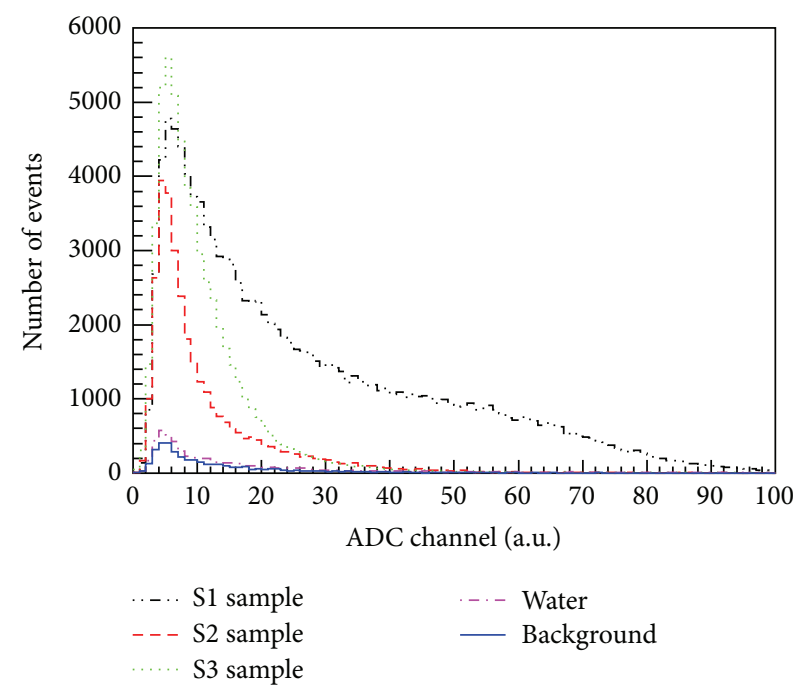

(a)

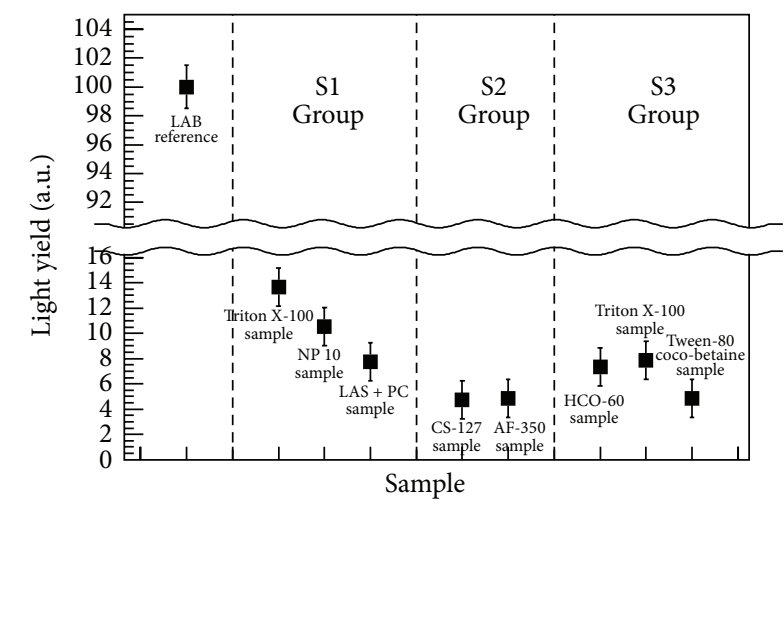

(b)

FIGURE 5: (a) Scintillation response from each sample. (b) Light yield (LY) of liquid scintillators using water. Results of some liquid scintillators in each group (S1, S2, and S3) with different surfactants are shown. The liquid scintillators using water are excited by using ${ }^{137} \mathrm{Cs}$ source. A LAB-based LS is used for a reference. We take a LAB-based LS as $100 \%$ for comparison with other samples.

3.3. Absorption and Fluorescence Emission Spectra. Based on the absorption spectra of the samples, excitation wavelength was selected. A fluorescence check of S1 (Triton X-100), S2 (CS-124), and S3 (HCO-60) sample was carried out by using a Varian Cary Eclipse fluorescence spectrophotometer over a wide spectral range of excitation wavelengths to look for unusual fluorescence in the wavelength region of
$350 \sim 450 \mathrm{~nm}$. The pure LAB solvent shows an emission maximum at $340 \mathrm{~nm}$. Therefore, the wavelength of the scintillation light from LAB needs to be shifted above $400 \mathrm{~nm}$. This can be done by adding a primary solute such as PPO and CS124. PPO emits photons at $340 \sim 440 \mathrm{~nm}$. The absorption and the emission spectra of S1 (Triton X-100), S2 (CS-124), and S3 (HCO-60) sample are shown in Figure 7. The results show 
TABLE 3: Density of S1, S2, and S3 sample group. A LAB-based LS is added for a reference sample in the measurement of density comparison.

\begin{tabular}{llccc}
\hline Number Sample group & Surfactant & $\begin{array}{c}\text { Density } \\
\left(\mathrm{g} / \mathrm{cm}^{3}\right) \\
\pm 0.001\end{array}$ & $\begin{array}{c}\text { Temperature } \\
\left({ }^{\circ} \mathrm{C}\right)\end{array}$ \\
\hline (1) & LAB-based LS & & 0.852 & 28.0 \\
\hline (2) & S1 & LAS & 0.998 & 26.1 \\
(3) & Triton X-100 & 1.011 & 25.2 \\
(4) & & NP-10 & 0.996 & 27.6 \\
\hline (5) & S2 (CS-124) & & 0.997 & 26.4 \\
(6) & S2 (AF-350) & & 0.996 & 27.6 \\
\hline (7) & & HCO-60 & 1.015 & 25.6 \\
(8) & S3 & Triton X-100 & 1.007 & 25.1 \\
(9) & & Polysorbate-80 \\
& & + coco-betaine & 0.995 & 27.0 \\
\hline
\end{tabular}
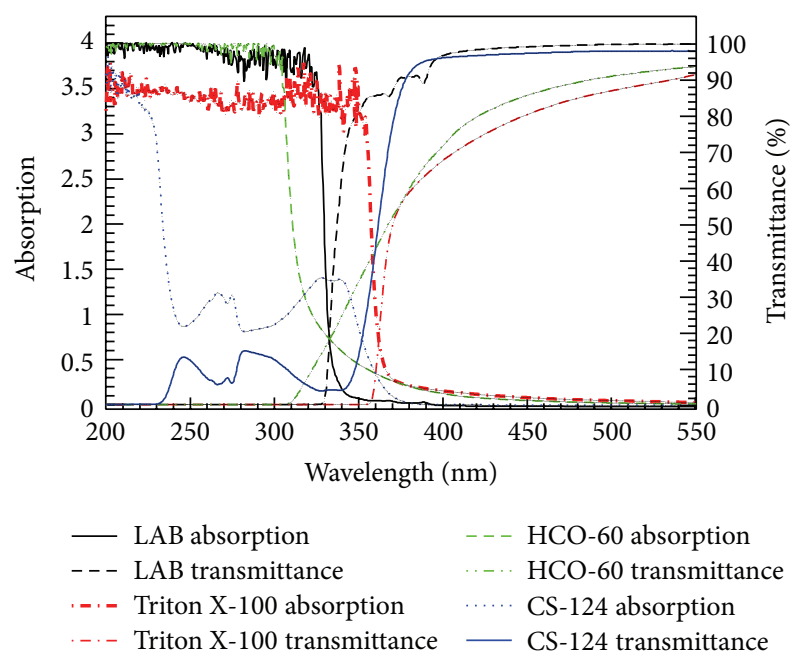

Figure 6: Transmittance $[T]$ and absorption $[A]$ values of $S 1$ (Triton $\mathrm{X}-100)$, S2 (CS-124), and S3 (HCO-60) samples as functions of wavelength on one plot. The solid line represents the absorption, and the dashed line represents the transmittance. $T$ is related to $A=-\log T$. The transmittance of various samples is given as a percentage.

that the emission spectrum of each sample is well matched with the absorption spectrum of each sample. The maximum value of the emission spectrum of each sample is located at about 420 430 nm and the Hamamatsu H6527 PMT had its optimal maximum quantum efficiency in this wavelength range. Therefore, the energy transfer mechanism of S1 (Triton $\mathrm{X}-100)$, S2 (CS-124), and S3 (HCO-60) samples is clearly seen.

3.4. Density. Generally a neutrino detector consists of several vessel layers, and each layer is filled with a different liquid. This causes different buoyancy forces between layers [23]. Therefore, it is better to know the density with reasonable accuracy. To minimize the mechanical stress, the density

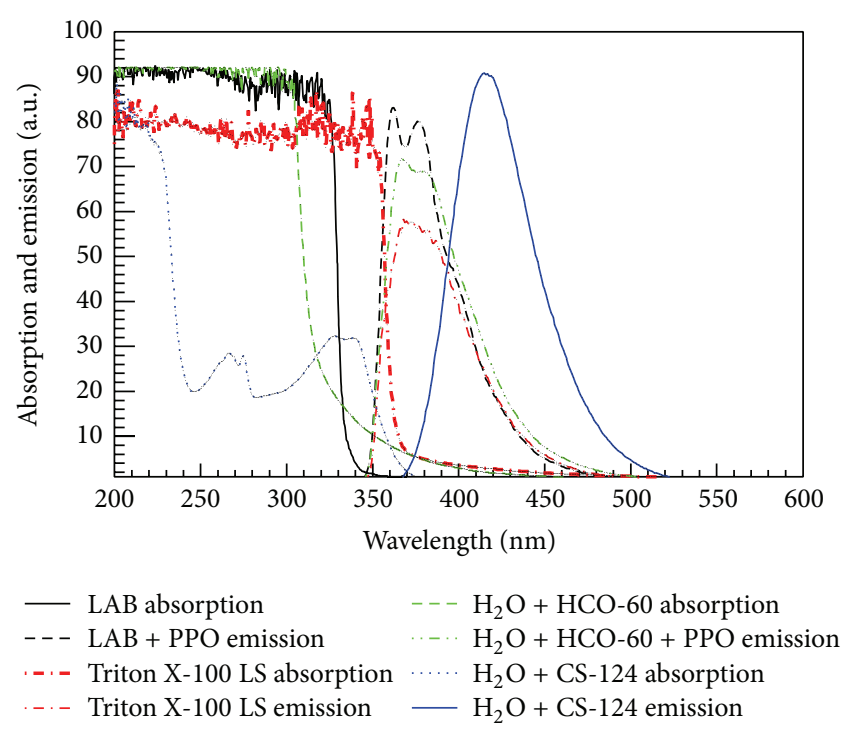

FIgURE 7: The absorption and the emission spectra of S1 (Triton $\mathrm{X}-100)$, S2 (CS-124), and S3 (HCO-60) samples, respectively. The overlap of absorption and emission spectra in each sample group can be seen.

of each layer needs to be matched as close as possible. We used a portable density meter (DA-130N, KEM) with a resolution of $0.001 \mathrm{~g} / \mathrm{cm}^{3}$ at $0 \sim 40^{\circ} \mathrm{C}$. The densities of S1, S2, and S3 samples we synthesized are shown in Table 3. For comparison, the density of a LAB-based liquid scintillator is $0.852 \pm 0.001 \mathrm{~g} / \mathrm{cm}^{3}$ at $28^{\circ} \mathrm{C}[24]$.

\section{Summary}

We have developed and established scintillating liquids using water as a solvent for an organic liquid scintillator. Understanding and characterizing the various properties of a liquid scintillator using water are important to use them. Several surfactants have been tested to solubilize the organic solvent in water. In particular, in this work we mainly focused on and investigated S3 samples in many ways, in which a fluor is added to water using different surfactants. Namely, we use a method where a fluor is directly dissolved in surfactants and then diluted with water.

A feasibility check for liquid scintillators using water shows that they have $\sim 10 \%$ of LY relative to that of a $100 \%$ LAB-based LS. This is a marginal LY to use. In addition, several important physical and optical parameters of the samples were measured and were satisfactory for use. The long-term stability test showed that three group samples were chemically stable over a year. A liquid scintillator using water with a nonionic surfactant has many good features to use for neutrino experiments. It can replace hundreds of tons of organic liquid scintillators and has relative simplicity of liquid handling and cost efficiency. It could potentially be a possible candidate for very large next generation neutrino detectors. 


\section{Conflict of Interests}

The authors declare that there is no conflict of interests regarding the publication of this paper.

\section{Acknowledgments}

This work was supported by the Korea Neutrino Research Center, which was established by the National Research Foundation of Korea (NRF) grant funded by the Korean government (MSIP) (no. 2009-0083526) and the Basic Science Research program through the National Research Foundation of Korea (NRF) funded by the Ministry of Education (2010-0007850, 2012-0001177, and 2012M2B2A6030210).

\section{References}

[1] B. Pontecorvo, "Neutrino experiments and the problem of conservation of leptonic charge," Journal of Experimental and Theoretical Physics, vol. 53, pp. 1717-1725, 1967.

[2] K. Inoue, "Reactor neutrino oscillation studies with KamLAND," New Journal of Physics, vol. 6, no. 1, pp. 147-170, 2004.

[3] P. K. Lightfoot, V. A. Kudryavtsev, N. J. C. Spooner, I. Liubarsky, R. Luscher, and N. J. T. Smith, "Development of a gadoliniumloaded liquid scintillator for solar neutrino detection and neutron measurements," Nuclear Instruments and Methods in Physics Research, Section A: Accelerators, Spectrometers, Detectors and Associated Equipment, vol. 522, no. 3, pp. 439-446, 2004.

[4] G. Alimonti, C. Arpesella, M. Balata et al., "Light propagation in a large volume liquid scintillator," Nuclear Instruments and Methods in Physics Research, Section A: Accelerators, Spectrometers, Detectors and Associated Equipment, vol. 440, no. 2, pp. 360-371, 2000.

[5] M. Yeh, A. Garnov, and R. L. Hahn, "Gadolinium-loaded liquid scintillator for high-precision measurements of antineutrino oscillations and the mixing angle, $\theta_{13}$," Nuclear Instruments and Methods in Physics Research A: Accelerators, Spectrometers, Detectors and Associated Equipment, vol. 578, no. 1, pp. 329-339, 2007.

[6] S. H. So, K. K. Joo, B. K. Kim, S. H. Song, and I. S. Yeo, "Investigation of the physical and the optical properties of various base solvents for the liquid scintillator in a neutrino detector," Journal of the Korean Physical Society, vol. 62, no. 1, pp. 26-33, 2013.

[7] J. S. Park, S. B. Kim, J. Lee, B. C. Kim, S. H. Kim, and K. K. Joo, "Feasibility study of a liquid scintillator using domestically produced Linear Alkyl Benzene (LAB)," New Physics, vol. 58, pp. 62-67, 2009.

[8] F. Elisei, F. Gatti, A. Goretti et al., "Measurements of liquid scintillator properties for the Borexino detector," Nuclear Instruments and Methods in Physics Research A, vol. 400, no. 1, pp. 5368, 1997.

[9] K. S. Park, J. S. Park, B. C. Kim et al., "Construction and properties of acrylic vessels in the RENO," Nuclear Instruments and Methods in Physics Research A, vol. 686, pp. 91-99, 2012.

[10] C. D. Shin and K. K. Joo, "Study on the neutrino oscillation with a next generation medium-baseline reactor experiment," Advances in High Energy Physics, vol. 2014, Article ID 320287, 8 pages, 2014.
[11] Y.-F. Li, "Overview of the Jiangmen Underground Neutrino Observatory (JUNO)," International Journal of Modern Physics: Conference Series, vol. 31, Article ID 1460300, 5 pages, 2014.

[12] Y. Fukkuda, T. Hayakawa, E. Ichihara et al., "Measurement of the solar neutrino energy spectrum using neutrino-electron scattering," Physical Review Letters, vol. 82, no. 12, pp. 24302434, 1988.

[13] P. B. Price, "Comparison of optical, radio, and acoustical detectors for ultrahigh-energy neutrinos," Astroparticle Physics, vol. 5, no. 1, pp. 43-52, 1996.

[14] R. Nahnhauer, "Alternative detection methods for highest energy neutrinos," Nuclear Physics B-Proceedings Supplements, vol. 143, pp. 387-394, 2004.

[15] I. S. Yeo and K. K. Joo, "Study on the optical properties of a linear alkyl benzene-based liquid scintillator in the RENO," New Physics, vol. 61, pp. 739-743, 2011.

[16] M. Yeh, S. Hans, W. Beriguete et al., "A new water-based liquid scintillator and potential applications," Nuclear Instruments and Methods in Physics Research A: Accelerators, Spectrometers, Detectors and Associated Equipment, vol. 660, no. 1, pp. 51-56, 2011.

[17] X. Dai, E. Rollin, A. Bellerive et al., "Wavelength shifters for water Cherenkov detectors," Nuclear Instruments and Methods in Physics Research, Section A: Accelerators, Spectrometers, Detectors and Associated Equipment, vol. 589, no. 2, pp. 290-295, 2008.

[18] D. R. Winn and D. Raftery, "Water-based scintillators for largescale liquid calorimetry," IEEE Transactions on Nuclear Science, vol. 32, no. 1, pp. 727-732, 1985.

[19] Y. W. Kuk, J. M. Lee, S. C. Cho, and S. D. Yeo, Colloid \& Surfactant, Dae Gwang Press, 2013.

[20] H. Nakahara, Contributions to the development of a waterbased neutrino detector for the T2K project [Ph.D. thesis], The University of British Columbia, 2006.

[21] I. R. Barabanov, L. B. Bezrukov, C. M. Cattadori et al., "Performances and stability of a 2.4 ton Gd organic liquid scintillator target for $\bar{v}_{\mathrm{e}}$ detection," Journal of Instrumentation, vol. 5 , no. 4 , Article ID P04001, 2010.

[22] J. S. Park, J. Lee, I. S. Yeo et al., "Production and optical properties of Gd-loaded liquid scintillator for the RENO neutrino detector," Nuclear Instruments and Methods in Physics Research, Section A: Accelerators, Spectrometers, Detectors and Associated Equipment, vol. 707, pp. 45-53, 2013.

[23] K. S. Park, J. S. Park, B. C. Kim et al., "Construction and properties of acrylic vessels in the RENO detector," Nuclear Instruments and Methods in Physics Research, Section A: Accelerators, Spectrometers, Detectors and Associated Equipment, vol. 686, pp. 91-99, 2012.

[24] B. C. Kim, S. W. Seo, and K. K. Joo, "Measurement of the density of liquid scintillator solvents for neutrino experiments," New Physics, vol. 61, no. 8, pp. 759-762, 2011. 

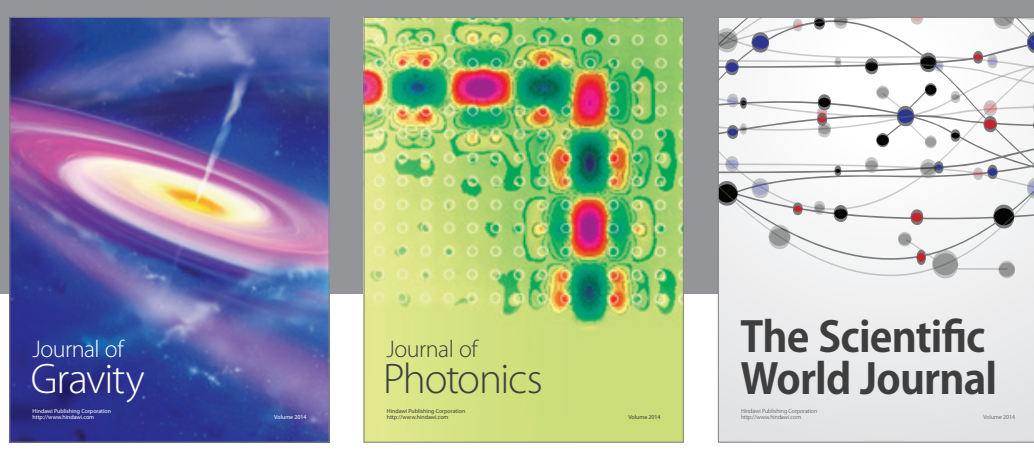

The Scientific World Journal
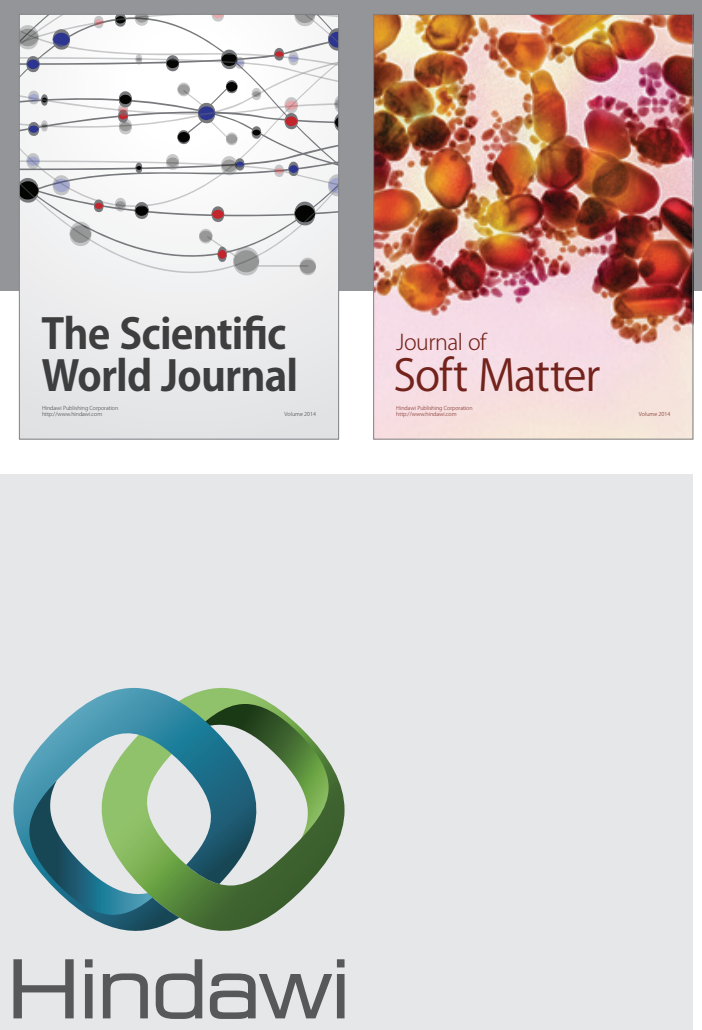

Submit your manuscripts at

http://www.hindawi.com

nternational Journal of

Statistical Mechanics
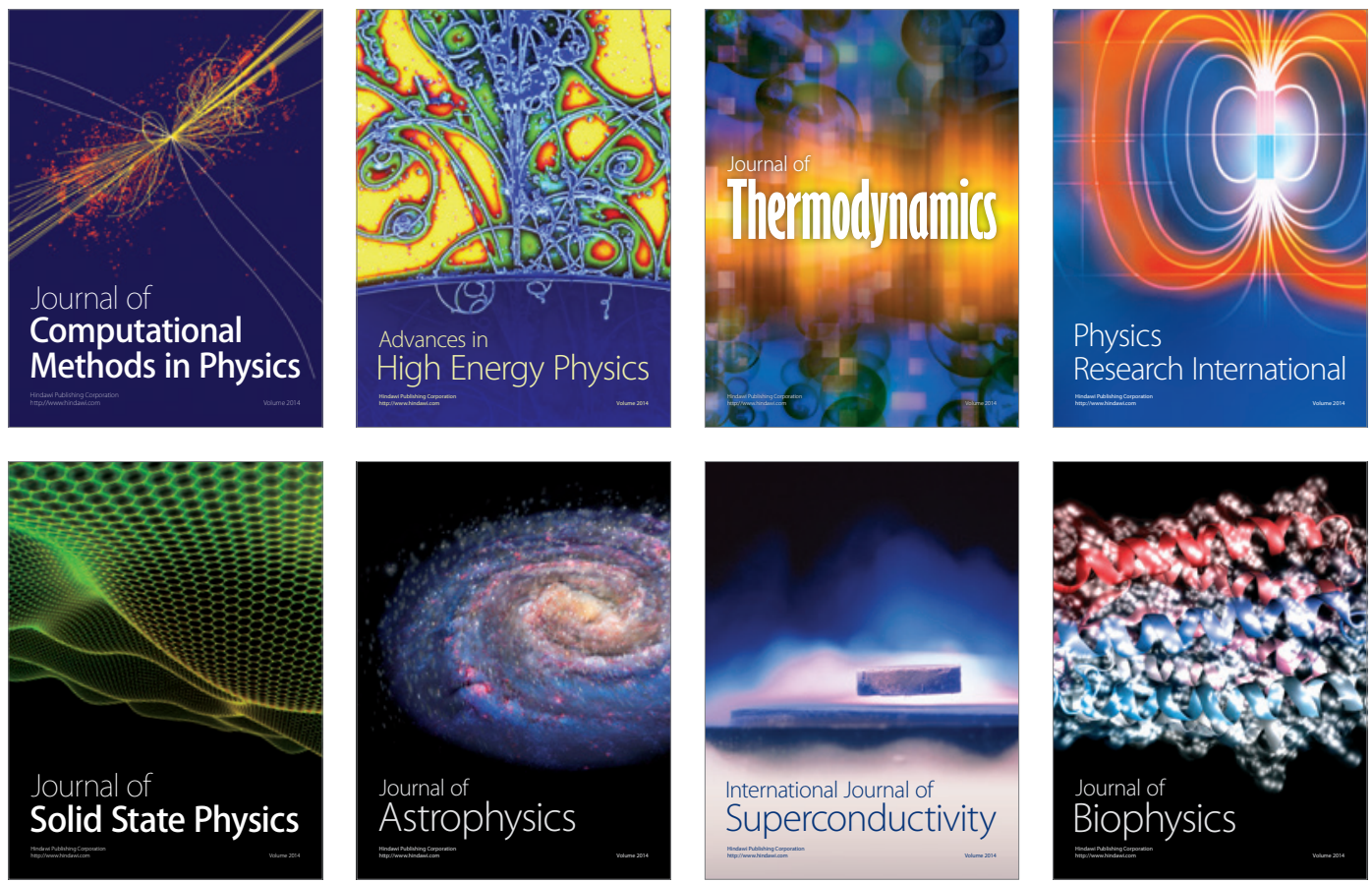
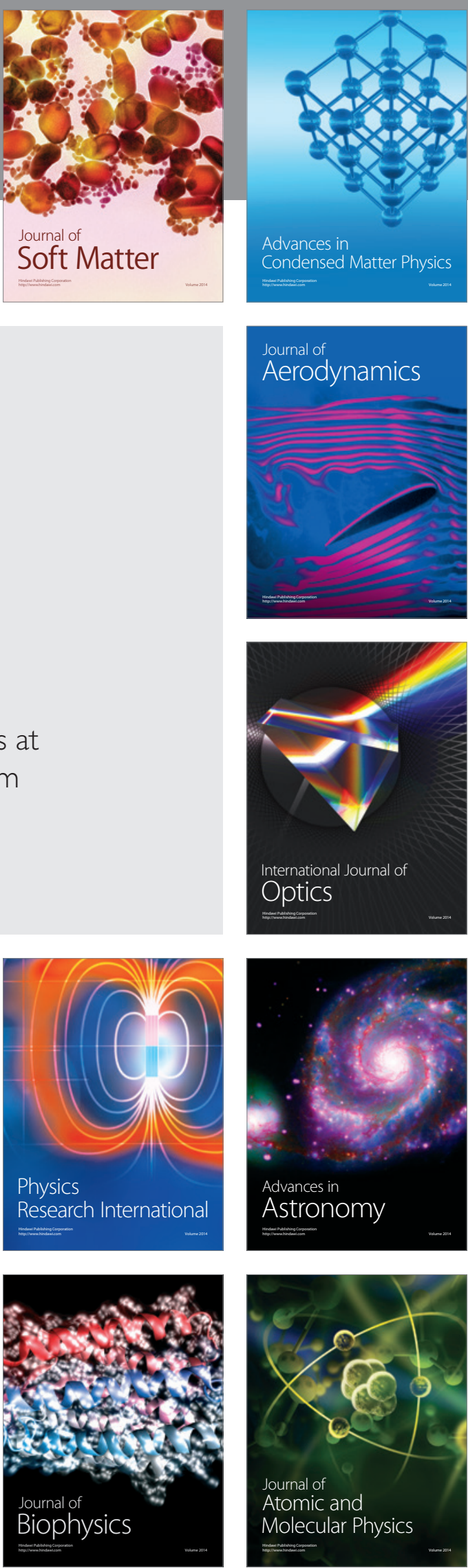